\title{
Philosophiques
}

\section{Esthétique et événement}

\section{Paradoxe et temporalité du sublime depuis Kant}

\section{Martin Mees}

Volume 43, numéro 2, automne 2016

URI : https://id.erudit.org/iderudit/1038212ar

DOI : https://doi.org/10.7202/1038212ar

Aller au sommaire du numéro

\section{Éditeur(s)}

Société de philosophie du Québec

ISSN

0316-2923 (imprimé)

1492-1391 (numérique)

Découvrir la revue

Citer cet article

Mees, M. (2016). Esthétique et événement : paradoxe et temporalité du sublime depuis Kant. Philosophiques, 43(2), 391-410. https://doi.org/10.7202/1038212ar
Résumé de l'article

Cet article propose une réévaluation de la notion d'« événement » en matière d'esthétique, ce qui nécessite de s'interroger plus spécifiquement sur la temporalité propre au concept de "sublime ", associé traditionnellement à une fulgurance, un instant sidérant qui ferait justement événement. Le développement s'appuie sur l'Analytique kantienne du sublime qui, en diverses occasions, met en avant le caractère paradoxal d'un sublime qui ne semble pouvoir se déployer qu'au prix d'une temporalité double, qualifiée ultimement de régressive. Je défendrai ici la thèse que le sublime renvoie en réalité à une expérience esthétique de l'événement comme tel, à même de nous faire penser une certaine " Idée esthétique » du temps. Une telle expérience semble d'ailleurs pouvoir trouver forme dans différentes productions poétiques de notre modernité. 


\title{
Esthétique et événement
}

\section{Paradoxe et temporalité du sublime depuis Kant}

\author{
MARTIN MEES \\ Université Saint-Louis - Bruxelles \\ Centre Prospéro - Langage, image et connaissance \\ martin.mees@usaintlouis.be
}

\begin{abstract}
RÉSUMÉ. - Cet article propose une réévaluation de la notion d' «événement» en matière d'esthétique, ce qui nécessite de s'interroger plus spécifiquement sur la temporalité propre au concept de «sublime», associé traditionnellement à une fulgurance, un instant sidérant qui ferait justement événement. Le développement s'appuie sur l'Analytique kantienne du sublime qui, en diverses occasions, met en avant le caractère paradoxal d'un sublime qui ne semble pouvoir se déployer qu'au prix d'une temporalité double, qualifiée ultimement de régressive. Je défendrai ici la thèse que le sublime renvoie en réalité à une expérience esthétique de l'événement comme tel, à même de nous faire penser une certaine «Idée esthétique» du temps. Une telle expérience semble d'ailleurs pouvoir trouver forme dans différentes productions poétiques de notre modernité.
\end{abstract}

ABSTRACT. - This article proposes a re-evaluation of the notion of "event" in the field of aesthetics, which requires to question the specific temporality of the concept of "sublime", traditionally associated with a flash, a staggering moment, which precisely constitutes an event. The development leans on the Kantian Analytic of the sublime which, on diverse occasions, highlights the paradoxical character of a sublime that only seems able to fulfil itself at the price of a double temporality, ultimately named regressive. I shall defend here the thesis that the sublime refers actually to an aesthetic experience of the event per se, allowing us to conceptualise a certain "aesthetic idea" of time. Such an experience, as a matter of fact, seems to be embodied in different poetic productions of our modernity.

Ainsi que de l'éclair, rien ne reste de l'heure Qu'au néant destructeur le temps vient de donner

[...] Et retenez-la bien au gré de votre envie, Comme le seul instant que votre âme rêva; Comme si le bonheur de la plus longue vie Était dans l'heure qui s'en va. Nerval, Le ballet des heures.

\section{Esthétique et événement}

D. Charles s'interrogeait, dans le Vocabulaire d'esthétique dirigé par E. Souriau, sur la pertinence du concept d' «événement» en matière d'esthétique. Il dégageait deux emplois possibles du terme. D'un côté, l'idée d'un événement esthétique au sens d'un événement artistique, catégorie sous laquelle il semble possible de ranger un certain nombre de dates et d'œuvres-clés de 
l'histoire de l'art. L'auteur rappelle la proposition de P. Francastel consistant à regrouper ces œuvres phares sous le nom de «Formes ", les distinguant par là du vaste domaine des formes. D'un autre côté, l'auteur avance l'idée que l'esthétique, au sens d'une étude de la sensibilité, n'a pas nécessairement à s'encombrer de l'œuvre, de son rapport aux catégories ou à la tradition de l'art, et que l'événement esthétique peut simplement s'entendre au sens du "particulier vécu avec intensité » (J. Wahl) qui peut parfaitement s'éprouver «dans mille activités humaines familières ou grandioses et jusque dans certaines démarches de la nature ${ }^{1} »$ (E. Souriau).

Il est intéressant de constater que ces conceptions de "l'événement esthétique » rappellent toutes deux un autre concept de la tradition philosophique: le sublime. Dans son acception classique, telle que définie par le Pseudo-Longin et Boileau à sa suite, le sublime renvoie en effet à une qualité particulière de l'art, un "plus-que-beau » que n'atteindraient que les génies anciens. Dans son traité Peri Hupsous, le Pseudo-Longin énumère les chefsd'œuvre du passé méritant le qualificatif de sublime, ces «Formes» dont doit s'inspirer l'artiste contemporain. La nécessaire partialité du critère invoqué pour trancher entre les œuvres du génie et le reste de l'art a, comme on le sait, poussé Burke et Kant à retirer le sublime du domaine des formes pour en faire un certain jugement esthétique de l'homme. Le sublime ne réside plus dès lors que dans la subjectivité du spectateur, et plus encore lorsque cette dernière se trouve face aux manifestations de la Nature que devant les objets de l'art. Le sublime est caractérisé par un effet profond, paralysant ou enthousiasmant, sur ceux qu'il touche, renvoyant bien à l'intensité de vécu évoquée ci-dessus. L'insistance des philosophies du sublime sur la spécificité et la force de son effet sur l'homme a mené plusieurs critiques récentes à considérer le sublime comme ce qui fit rupture par rapport à la poétique classique, marquant l'avènement de l'esthétique pour qui prime la réception du spectateur sur les conditions de production de l'œuvre. Le sublime, en ces différents sens, cristallise donc tout ce qu'on peut entendre dans l'idée d'événement esthétique, allant même jusqu'à représenter pour certains l'événement même de l'esthétique².

\section{Le sublime comme événement}

Événement de l'art ou événement de l'esthétique, le sublime semble traduire un surgissement: depuis le traité du Pseudo-Longin, il est en effet associé à

1. E. Souriau (dir.), Vocabulaire d'esthétique, Paris, Presses Universitaires de France, réed. 2010, p. 738 .

2. J.-L. Nancy insiste sur ce point: «Ce que la tradition nous transmet sous le nom de "sublime" n'est pas une esthétique (surtout pas telle ou telle esthétique du grandiose, du monumental ou de l'extatique, avec quoi le sublime est souvent confondu [...]. La tradition nous transmet l'esthétique comme question.» J.-L. Nancy, "Introduction», in J.-F. Courtine, M. Deguy, et al., Du sublime, Paris, Belin, 2009, p. 5-6. 
l'image de l'éclair, agissant de manière fulgurante, nous saisissant tout en demeurant lui-même insaisissable. Mais qu'est-ce qui se donne à nous dans cette fulgurance? Il semble que ce ne soit rien de moins qu'un certain dévoilement de «sens».

De l'Antiquité à certaines théories contemporaines du sublime (Lyotard, Derrida, Nancy, Lacoue-Labarthe, Richir, etc.) se développe une compréhension du sublime - plus ou moins explicite selon les cas - comme un absolu, la plupart du temps indicible, incommensurable, hébergeant une vérité dont le dévoilement (repris de la figure d'Isis), toujours partiel et inachevé, constitue le point culminant de l'expérience du sublime. Dans cette perspective, le sublime est hypostasié tout en étant ramené à une figure négative: le silence, l'imprésentable, le «maintenant » par définition insaisissable, la limite elle-même, le phénomène "pur». Le sublime est pensé comme l'audelà de l'art, le fil ténu de sa clôture alors même qu'il tente d'outrepasser son domaine à l'impossible 3 . Hegel dira: "Le sublime, en général, est la tentative d'exprimer l'infini sans trouver dans le domaine des apparences visibles un objet capable de le représenter. ${ }^{4}{ }^{»}$

Ce qui semble certain en ce qui concerne les définitions traditionnelles du sublime, c'est que quelque chose survient en un éclair et s'empare de notre entendement comme de notre sensibilité, puisque le sublime est l'émotion-limite, indescriptible et ambiguë, qui accompagne la révélation d'une vérité supra-sensible. L'apparition de cette vérité ne se fait pas sur un mode discursif, elle est là soudain entière, absolue et donc indicible. Si le sens luimême fait toujours "événement", comme l'affirment plusieurs penseurs de ces dernières décennies, le sublime en serait l'expérience, disons même son appréciation esthétique. Si l'on peut dire que le sublime est événement esthétique, c'est en ce qu'il représenterait la possibilité même qu'un sens survienne de la rencontre de l'œuvre et de l'homme. Cette conception du sublime semble néanmoins problématique: en disant cela, on n'a pas encore dit grand chose. Quel est ce sens qui apparaît, quelles sont les conditions de l'expérience du sublime? Comment s'opère concrètement ce dévoilement? Le sublime a trop souvent servi une conception de l'art volontairement abstraite, obscure ou mystique, déjà dénoncée par Hegel qui choisit en conséquence d'abandonner ce concept.

Il semble néanmoins qu'une approche plus circonstanciée de l'idée du sublime permettrait de conserver l'intérêt intrinsèque à cette notion - qui continue, malgré la disqualification hégélienne, à interroger aujourd'hui

3. La philosophie du sublime fait de son objet la propension de l'art à dire quelque chose qui n'est pas l'art, " un art qui tremble au bord de l'art, se donnant pour tâche autre chose que l'art, $[\ldots]$ quelque chose de "sublime" [...] disons la vérité, ou l'expérience [...]». J.-L. Nancy, "L'offrande sublime", in J.-F. Courtine, M. Deguy, et al., Du sublime, op. cit., p. 47.

4. G. W. F. Hegel, Esthétique; B. Timmermans et P. Zaccaria (dir.), Paris, Le Livre de poche, I997, vol. I, p. 470. 
notre rapport à l'art ${ }^{5}$ - sans pour autant servir une de "ces esthétiques implicites, inavouées ou naïves ", dénoncées par L. Van Eynde, "qui accordent à l'œuvre d'être le lieu mystérieux de l'épiphanie d'un sens incommensurable à toute articulation discursive, à toute autre production de sens, notamment conceptuelle ${ }^{6}$.» Or cette autre approche passe peut-être par une reconsidération de la dimension "événementielle» du sublime, de l'assimilation implicite du concept avec l'idée d'un événement lui-même compris de manière caricaturale: moment d'exception, surgissement, rupture dans l'ordre du sens. J.-F. Lyotard invoque par exemple à propos du sublime, comme le rappelle D. Vienet, "le plaisir "d'accueillir l'inconnu", la joie spinozienne procurée par "l'accroissement d'être qu'apporte l'événement" ." Dans son analyse de l'expérience du sublime, M. Baudin souligne également ce trait, associé à l'idée d'un sublime comme expérience esthétique forte: "[il est] ce qui s'éprouve, ce qui venant de la rencontre avec un événement, c'est-à-dire un inattendu, tient de la mise à l'épreuve; autrement dit: de la déstabilisation des équilibres antérieurs, voire de leur mise en danger, ou de leurs remaniements ${ }^{8}$ ». Une telle acception de l'événement, quelque peu simpliste ou univoque, aurait comme conséquence de cautionner une théorie du sublime laissant son objet à l'état d'abstraction imprécise si ce n'est indicible. Peut-être, en examinant le rapport du sublime au temps de manière plus approfondie, pourrait-on faire droit à une conception plus riche de l'événement qui, du même coup, «déshypostasierait» le sublime en nous forçant à l'inscrire dans le temps, à préciser la temporalité de son expérience et de son sens. Même si Emmanuel Kant ne traite pas systématiquement de la dimension temporelle du sublime, il semble néanmoins, à la lecture de sa troisième Critique, que différents éléments de son analyse soient particulièrement pertinents pour mener une telle étude, qui vise d'ailleurs moins à rendre fidèlement compte du jugement kantien qu'à réinvestir le concept du sublime depuis un axe qui semble fécond: l'événement.

5. Nous pouvons citer quelques titres de manière non-exhaustive: Jean Bessière, "Le sublime aujourd'hui: d'un discours sur le pouvoir de l'art et de la littérature, et de sa possible réécriture ", La littérature et le sublime, 2007; Denis Viennet, "Il y a "quand même". À propos du sublime aujourd'hui ", Philosophique, 20I I Michael Shapiro, "The Sublime Today: RePartitioning the Global Sensible ", Millennium, 2006; Glenn W. Most, "The Sublime, Today ? ", Dynamic reading, 20I2; Gillian B. Pierce, The Sublime Today: Contempory Readings in the Aesthetic, Cambridge Scholars Publishing, 20I2; Jeremy Gilbert-Rolfe, Das Schöne und das Erhabene von heute, Merve Verlag Berlin, 1996.

6. L. Van Eynde, "Une utopie: l'institution littéraire de la société ", in J.-P. Madou, S. Santi et L. Van Eynde (dir.), Mythe et création 2. L'œuvre, l'imaginaire, la société, Chambéry, Presses de l'Université de Savoie, 2007, p. 33.

7. D. Viennet, "Il y a "quand même". À propos du sublime aujourd'hui ", Philosophique [En ligne], consulté le 29-IO-20I 5, disponible sur [http://philosophique.revues.org/I7I], p. 66.

8. M. Baudin, «L'expérience du sublime ", Topique, 2009, 4, n I09. 


\section{La temporalité du sublime}

À lire Kant, le sublime se trouverait, à première vue, du côté d'un événement entendu comme rupture, discontinuité, tandis que le beau se caractériserait par la continuité, la durée. En effet, nous dit l'Analytique du sublime, «le goût pris à ce qui est beau présuppose que l'esprit soit dans un état de calme contemplation et l'y maintient ${ }^{9}{ }^{\prime}$. Le beau s'épanouit dans l'harmonie des facultés, et le plaisir qu'il engendre est un plaisir qui dure, sans interruption. Le sublime, quant à lui, est fugitif: on ne peut retenir ce sentiment qui dérange et met en mouvement l'esprit. "Ce mouvement (en son début particulièrement) peut être comparé à un ébranlement, c'est-à-dire à la rapide succession de la répulsion et de l'attraction par un même objet ${ }^{10}$." Cet ébranlement marque une rupture dans le quotidien et se compose lui-même de différentes "phases» qui tranchent les unes sur les autres. Aussi le plaisir du sublime naît-il lui-même "de la cessation d'une situation pénible ${ }^{11}$ ". L'affaire semble entendue et irait dans le même sens que les précédentes observations: il y aurait d'un côté la calme contemplation du beau, de l'autre l'ébranlement du sublime qui surgit, fait rupture et s'évade. Le philosophe de Königsberg paraît reprendre à son compte l'idée d'un sublime-événement tel qu'ont pu le décrire ses précurseurs et que le décriront ses héritiers. Chez lui cependant, l'ambiguïté inhérente au sublime (qui se décline en joie et en peine) sera marquée temporellement: "Une rapide succession de la répulsion et de l'attraction ». Comment se concilient l'idée d'une succession et celle d'une fulgurance?

Kant avance l'idée que la succession d'états contraires lors de l'expérience du sublime ne produit aucun décalage temporel: elle se situe toute entière dans une simultanéité immédiatement appréhendée par notre faculté de juger. De là découle le double effet du sublime, la coïncidence d'un sentiment de finitude, d'impuissance de notre imagination à se représenter les Idées de la raison, et du sentiment d'une destination supérieure de l'homme, «supériorité de la destination rationnelle de notre faculté de connaître sur le pouvoir le plus grand de notre sensibilité12». Kant marque explicitement cette simultanéité: "Notre imagination [...] prouve ses bornes et son impuissance, mais en même temps aussi sa destination qui est la réalisation de son accord avec cette Idée comme avec une $\operatorname{loi}^{13}$.» La conciliation que nous cherchions s'opère donc dans un certain paradoxe: le sublime fait advenir «en même temps» une chose et son contraire, puissance et impuissance,

9. I. Kant, Kritik der Urteilskraft, hrsg. von der Königlich Preußischen Akademie der Wissenschaften $(A k)$, Bd. V, Berlin, I908, 247. Trad. fr. A. Philonenko, Paris, Vrin, 2000, p. I22. Nous soulignons.

10. $A k, \mathrm{~V}, 258$. Trad., p. 138 .

11. Ak, V, 26I. Trad., p. I42.

12. $A k, \mathrm{~V}, 257$. Trad., p. I37.

13. Ak, V, 257. Trad., p. I36-I37. Nous soulignons. 
plaisir et douleur. C'est à ce seul prix que semble pouvoir se maintenir l'instantanéité d'un sublime décrit encore comme médiat et immédiat. Le sublime agit en un instant étant "ce qui plaît immédiatement par la résistance qu'il oppose à l'intérêt des sens ${ }^{14}$ ». Mais il est aussi médiat, en ce qu'il ne vaut pas pour lui-même, contrairement au beau pour lequel suffit la qualité formelle de l'objet visé: "Le sublime consiste seulement en la relation, en laquelle le sensible de la représentation de la nature est jugé propre pour un usage supra-sensible possible de celui-ci ${ }^{15}$.»

Si le sublime est un événement, et si l'événement est intrinsèquement lié à l'émergence d'un sens - thèse défendue depuis Aristote jusque Deleuze ${ }^{16}$ - c'est par le caractère paradoxal du sublime que peut se comprendre le sens accompagnant éventuellement son expérience. Le paradoxe, par définition, possède une force qui relève de la surprise, du saisissement, de l'étonnement, et qui ouvre les bornes de notre imagination en nous présentant une forme intuitionnable de manière immédiate, car condensée dans la tension d'une association contradictoire, qui demeurait jusque là inanticipée. Le sublime, dont l'événement semble placé sous le signe du paradoxe, jouit de la potentialité propre à ce dernier de créer de la nouveauté, de faire advenir de l'inattendu, de réaliser ce qui demeurait hors de tout champ d'attente. Cette idée n'est pas neuve: depuis le Pseudo-Longin, le sublime est pensé comme le paradoxon, ce qui semble ne pouvoir se présenter et qui pourtant se présente, selon la définition de P. Lacoue-Labarthe:

To paradoxon: cela, normalement, ne se traduit pas. Je traduirai cependant: das Unheimliche, pensant évidemment à l'usage heideggérien du mot; mais aussi à cette célèbre définition de Schelling: "On appelle unheimlich tout ce qui devrait rester secret, voilé, et qui se manifeste ${ }^{17}$."

Lacoue-Labarthe fait bien sûr allusion à la sentence d'Isis, où la déesse énonce elle-même son indicibilité, où elle dévoile l'impossibilité de tout dévoilement: "La sentence est laissée à sa paradoxale énigme, celle qui engendre, non la jubilation, mais un frisson sacré. La vérité en son essence est non-vérité18 ${ }^{18}$ "Selon Kant, les objets sublimes «nous font découvrir en

14. Ak, V, 267. Trad., p. I 50 . Nous soulignons.

15. Ak, V, 266-267. Trad., p. I49. Nous soulignons.

16. Aristote déjà, à travers l'idée de Tuchè (fortune, hasard), fait de l'événement la condition de possibilité d'une indétermination fondatrice, sur fond de laquelle va pouvoir se déployer le champ du possible. Plus récemment, Deleuze fait de l'événement la forme par excellence de l'émergence d'un sens, qui arrive de manière impersonnelle, non intentionnelle, mais éprouve l'homme dans sa capacité à l'accueillir, à se porter à sa hauteur. En des sens certes très distincts, les différentes pensées de l'événement traduisent chacune une ouverture dans l'horizon d'attente du sujet qui en fait l'expérience.

17. P. Lacoue-Labarthe, "La vérité sublime», in J.-F. Courtine, M. Deguy, et al., Du sublime, op. cit., p. I 86.

18. Ibid., p. I6r. 
nous un pouvoir de résistance d'un tout autre genre ${ }^{19}$ ", produisant une "satisfaction relative à l'extension de l'imagination elle-même ${ }^{20}$ ", puisque le sublime "éveille le sentiment d'une faculté supra-sensible en nous", "une faculté de l'âme qui dépasse toute mesure des sens ${ }^{21}{ }^{2}$. Cette ouverture ne se fait pas dans la douceur agréable du beau. Kant parle d'une «violation» de l'imagination, qui n'augmente ses perspectives qu'au prix de la douleur résultant de l'inadéquation de la sensibilité avec la raison. Les objets qui agrandissent le plus les bornes de notre imagination sont donc considérés par le philosophe comme les plus sublimes, et on ne s'étonnera pas qu'ils opèrent justement par la puissance du paradoxe. Serait sublime, par exemple, un homme qui, malgré sa faiblesse, ou la perspective de sa mort, ne plierait pas. Ou encore un guerrier qui parviendrait à faire preuve de douceur. De même, comme le montrait avec justesse Lacoue-Labarthe, les exemples textuels que Kant donne du sublime - particulièrement la sentence d'Isis relèvent également d'une logique profondément paradoxale. C'est donc par la force du paradoxe, et même à sa seule condition, que s'épanouit un sublime considéré comme événement esthétique. Si la Critique de la faculté de juger a bien une dimension "doxologique ", pour reprendre l'expression d'A. Philonenko $\mathrm{ko}^{22}$, s'attaquant notamment à la doxa et au sens commun, il semble bien que le sublime représente comme le revers de son objet, adoptant une structure qui relève du "para-doxal».

\section{Un temps du paradoxe}

Que pourrait être l'événement paradoxal du sublime? Pour tenter de répondre, il faut opérer un détour par l'idée de limite. Cette dernière, qui participe à la définition kantienne du sublime et que celui-ci travaille en retour, ne renvoie pas seulement à une dimension spatiale dans le cas qui nous occupe, mais prend sens aussi dans le rapport du sublime à la temporalité. En nous faisant prendre conscience d'une limite en nous (celle de notre imagination, de notre capacité de représentation), le sublime amène l'imagination à la conscience de l'au-delà de la limite, non sur le mode d'un connaître, mais par un jugement.

Or cette idée du supra-sensible [...] nous ne la connaissons pas, mais nous pouvons seulement la penser et elle est évoquée en nous par un objet dont le jugement esthétique étend l'imagination jusqu'à ses limites, soit en extension [mathématique] soit selon sa force sur l'esprit [dynamique], puisqu'il se fonde

19. Ak, V, 26I. Trad., p. I42.

20. Ak, V, 249. Trad., p. I26.

21. Ak, V, 250. Trad., p. I 27.

22. A. Philonenko, "Science et opinion dans la Critique de la faculté de juger", in M. Franck, J.-P. Larthomas et A. Philonenko, Sur la Troisième Critique, Combas, Éditions de l’Éclat, I994. 
sur le sentiment d'une destination de celui-ci [l'esprit], qui dépasse complètement le domaine de l'imagination ${ }^{23}$.

Cet au-delà, ce qui donc, par principe, est illimité, et qui correspond dans la théorie kantienne aux Idées de la raison, c'est ce que doivent pouvoir présenter les objets dits "sublimes", fût-ce négativement. "La nature est ainsi sublime dans ceux de ses phénomènes, dont l'intuition suscite l'idée de son infinité ${ }^{24}$.» D'une conception du sublime comme événement marquant une rupture (celle du sens, celle de l'appréciation esthétique, celle de l'instant incommensurable), nous pouvons légitimement passer à une conception de l'événement comme limite, moment de condensation paradoxal du limité mais aussi de l'illimité, donc de la rupture et de la continuité. Aussi peut-on affirmer que le sublime renvoie à une notion dialectisée de l'événement, qui seule peut rendre compte du rapport paradoxal que ce dernier entretiendrait à la temporalité. Comme le développe H. Gueguen,

Le modèle de l'événement recourt à un autre type de temporalité: un temps qui n'est plus, comme le soulignent Deleuze ainsi que Romano, le temps de l'expérience ordinaire et quotidienne (succession et différentiation du passé, du présent et du futur), mais un temps inassignable et non datable. [...] l'aiôn (en grec: le perpétuel, le "toujours") et qu'il décrit comme un "temps mort", un "entre-temps", "toujours déjà passé et éternellement encore à venir"25.

C'est un tel temps paradoxal que le sentiment du sublime amène à la conscience, non un «hors temps » complet, mais un décalage du temps par rapport à lui-même. Cela ne s'actualise pas sans certaines conséquences sur l'expérience du sublime elle-même, conséquences qui transparaissent à divers endroits de l'Analytique du sublime. Le sujet ne parvient pas à appréhender en un tout les diverses facettes de son sentiment: le paradoxe marque la dimension insaisissable du sublime, qui n'est plus indicible du fait de sa fulgurance, mais l'est, au contraire, par son inscription dans une temporalité double.

Dans cette opération les premières perceptions s'évanouissent toujours en partie avant que l'imagination ne saisisse les dernières, et la compréhension n'est jamais parfaite. [...] [E]n ceci l'imagination atteint son maximum et dans l'effort pour le dépasser, elle s'abîme en elle-même, et ce faisant est plongée dans une satisfaction émouvante ${ }^{26}$.

23. $A k, \mathrm{~V}, 268$. Trad., p. I 52. Nous soulignons.

24. $A k, \mathrm{~V}, 255$. Trad., p. I 34 .

25. H. Gueguen, "Du possible à l'événement: essai de typologie à propos de l'événement et de la contingence", Nouvelle revue de psychosociologie, 2015/I (n ${ }^{\circ}$ 19), p. 88. Chez Deleuze, le chronos représente une infinité de points qui se succèdent, tandis que l'aiôn est lui un point qui reprend l'infinité. Cette seconde définition correspond tout à fait à la dynamique temporelle présente dans le sublime.

26. Ak, V, 252. Trad., p. I3O. 
Le sublime, comme présentation simultanée d'une succession, va être associé à une temporalité spécifique, pouvant rendre compte de son paradoxe. Il s'agira d'une temporalité opérant à l'inverse de la progression chronologique qui traduit le mouvement objectif de l'imagination, mesurant et mettant en forme l'espace de façon discursive. Une temporalité, donc, que Kant nommera "régressive» dans un passage qui n'a été que trop rarement mis en exergue par la critique:

La compréhension de la pluralité dans l'unité, non de la pensée, mais de l'intuition, par conséquent la compréhension du successivement saisi en un instant [des Sukzessiv-Aufgefaßten in einen Augenblick] est, en revanche, une régression [ein Regressus] qui inversement supprime la condition temporelle dans le progrès de l'imagination [die Zeitbedingung im Progressus der Einbildungskraft wieder aufhebt] et rend intuitionnable la coexistence [das Zugleichsein anschaulich macht]. Aussi bien est-ce (puisque la succession temporelle est une condition du sens interne) un mouvement subjectif de l'imagination, par lequel elle fait violence au sens interne ${ }^{27}$.

À nouveau, la découverte du paradoxe est associée à une violence, faite ici à notre appréhension coutumière d'un temps "progressif », celle-ci se trouvant renversée dans l'expérience du sublime. Le paradoxe engendre une tension interne, que Kant nomme un "conflit des puissances ", qui explique la violence présente au sein de l'expérience du sublime, elle qui crée un «étonnement, qui confine à l'effroi, l'horreur et le frisson sacrée ${ }^{28} »$. Or il semble que la régression à l'œuvre dans le sublime s'accompagne d'un retour de la conscience sur elle-même, d'un mouvement régressif à comprendre comme «réflexif», précisément "ce qui rend intuitionnable» la coexistence, das Zugleichsein, littéralement «l'être en même temps». L' 'étonnement» engendré n'est pas étranger à une telle opération dans l'expérience du sublime, il n'est pas que surprise, mais aussi déprise de soi. C'est le moment où s'interrompt le fil du quotidien, le "progrès de l'imagination », le moment où se suspend la conscience intentionnelle. Cet aspect de l'étonnement est le même que celui du thaumazein grec, qui initie la réflexion philosophique par un étonnement premier du monde. À son image, le jugement esthétique du sublime invite à une certaine réflexivité de l'homme sur sa propre expérience, où semble être mise en cause l'idée même du temps. Car le sublime n'est pas seulement un instant insaisissable faisant rupture dans le quotidien, sa structure profondément paradoxale va nous forcer à penser une temporalité à sa mesure, une temporalité elle-même paradoxale, en ce qu'elle tranche sur l'habitude et propose un modèle «inverse ", capable de combiner des contraires.

27. Ak, V, 258-259. Trad., p. I39. Nous soulignons.

28. Ak, V, 269. Trad., p. I 53 . 


\section{Une esthétique de l'événement}

Comment comprendre cette temporalité régressive? Kant emploie le latin regressus (retour, marche rétrograde) plutôt que les mots allemands signifiant la régression, sans doute pour accentuer la spécificité de ce mouvement. Il s'agit de prendre le temps «à rebrousse-poil », non pas remonter son cours, bien entendu, mais prendre conscience de son action, de son mode de déploiement, qualifié de "progressif », et du rapport spécifique que l'homme entretient avec lui. Il faut y voir plus encore que la pose conjointe du temps et de son négatif: il s'agit bien d'aller à rebours de la "chrono-logique» en faisant place à un mode de présentation qui excède ce modèle et qui relève du «para-doxe» (étymologiquement «contre l'opinion» mais traduisible aussi par «contre toute attente»). Cette régression est un mouvement, terme que Kant mobilise abondamment pour caractériser le sublime: non pas simplement une perspective ou un "coup d'œil » instantané, mais un effort luimême inscrit dans une certaine durée. C'est dans ce mouvement que se comprend l'idée d'une dynamique du sublime, qui met en tension et ébranle le sujet inscrit dans une certaine temporalité. Un mouvement dont le sublime est le moteur, non plus uniquement «limite» mais véritable point de basculement concentrant simultanément une unité (finie) et une infinité. Cette idée du sublime va à l'encontre d'une conception de ce dernier comme inaccessible absolu ou présentation de l'imprésentable: l'au-delà de la limite ou son en-deçà. Le mouvement de basculement s'oppose à une conception de la limite comme frontière, ligne de partage fixe: si le sublime est limite, ce ne peut être que comme limite basculante, faisant passer le sujet qui en fait l'expérience d'un bord à l'autre de ce qu'elle prétendait circonscrire. Le "sub-lime» est étymologiquement ce qui travaille la limite, par le haut comme par le bas en fonction du sens attribué au sub latin. Il semble que ce ne soit pas simplement l'expérience $d u$ sublime qui soit traversée de sentiments antagonistes, mais que son jugement esthétique, le sublime comme concept, adopte une structure double. Nous rejoignons ici J.-L. Nancy lorsqu'il définit, de manière assez obscure à première vue, le sublime comme "cette motion par laquelle, incessamment, l'illimité s'enlève, s'illimite, le long de la limite qui se délimite et qui se présente ${ }^{29}$ ».

Nous nous éloignons ici de la théorie kantienne pour avancer une interprétation plus générale de cet événement du sublime. Ce dernier ne consiste donc pas en l'apparaître d'une quelconque limite figée, mais dans la non-résolution paradoxale d'une tension basculante. Dans le paradoxe est affirmée, non une équivalence mais une identité entre deux contraires qui ne coexistent pas réellement: l'un est toujours déjà l'autre, et vice-versa. Il n'y a pas à proprement parler et peine et plaisir, mais une peine qui est déjà plaisir et un plaisir qui est toujours souffrance. De même, l'instant sublime

29. J.-L. Nancy, «L'offrande sublime», op. cit., p. 65-66. 
renvoie toujours déjà à l'infini du temps, qui lui-même ne peut s'appréhender que dans l'unité de l'instant. Dans cet aller-retour proprement infini, aucune limite n'est traçable qui séparerait une chose de son contraire: nous pouvons seulement saisir une dynamique de l'ordre du basculement, d'une chose à l'autre et réciproquement, qui anime le sublime et dessine l'horizon de son infinité. Le sublime n'est pas ce qui a basculé, mais le basculement, cette opération, ce lien qui lie un endroit à son envers. Là réside sans doute la difficulté propre à l'expression du sublime, qui résiste à toute fixation, à toute immobilisation. Point de basculement, il incarne comme la part active de la limite, le mouvement réglant son éternel dépassement.

Pour résumer, nous pouvons certes dire que le sujet qui fait l'expérience du sublime bascule dans et par celle-ci: il y a bien une immédiateté, une fulgurance doublée d'une intensité qui fondent les caractéristiques traditionnelles du sublime. Ce basculement, comme nous l'avons montré, s'opère sans transition, il n'y a pas de limite qui serait un moment franchie. Schiller, développant la théorie kantienne, marque ce trait avec insistance:

Ainsi le sublime nous offre une issue pour franchir les bornes de ce monde sensible, où le sentiment du beau voudrait bien à tout jamais nous retenir prisonniers. Ce n'est pas peu à peu, (car entre la dépendance absolue et la liberté absolue il n'y a pas de transition possible), c'est soudain, et par une secousse, que le sublime arrache notre nature spirituelle et indépendante aux liens où la retenait la sensibilitée ${ }^{30}$.

Ainsi que le remarque également $\mathrm{M}^{\text {me }}$ de Staël, le sublime lui-même ne s'atteint pas par degrés, il n'est pas une limite ultime dont on pourrait s'approcher. Il ne peut être apprécié sur une échelle d'intensité: il n'y a aucun sens à parler d'un "très sublime » ou d'un "peu sublime ", on est dans le sublime ou non. Le sublime raté tombe dans son inverse et ne régresse pas en intensité: "Le sublime approché, c'est du sublime manqué. Et le sublime manqué c'est du risible ${ }^{31}$ ", comme le note E. Souriau. Pour autant, le sublime ne se maintient pas, il ne s'inscrit dans aucune durée dont nous pourrions prendre mesure. Baldine Saint Giron résume avec une grande justesse: « Le paradoxe du sublime est de se dérober au moment où il empoigne. Il prend à lui, surprend, et déprend de soi ${ }^{32}$." Le basculement, poursuivant son mouvement, retourne à son état premier en même temps qu'il retourne réflexivement l'homme sur lui-même. Le basculement émotionnel du sujet soudain pris d'un sentiment intense se double d'un basculement de sa conscience, qui ressent un basculement de la peine en plaisir, de l'impuissance en puissance, et vice versa. Le basculement en amène un autre qui est saisi par une réflexivité elle-même engendrée par ce mouvement.

30. F. Schiller, Du Sublime, trad. fr. A. Régnier, Arles, Sulliver, 2005, p. 22.

31. E. Souriau, "Le sublime ", Revue d'esthétique n I9, I966, p. 266.

32. B. Saint-Girons, Le sublime de l'Antiquité à nos jours, Paris, Desjonquères, 2005, p. I $8 \mathrm{I}$. 
En outre, la particularité du sublime dans le champ de nos expériences et son caractère nécessairement fulgurant n'impliquent pas nécessairement sa rareté. De la même façon que sublime et expérience du sublime sont intimement liés, l'infini que dévoile le sublime à la conscience caractérise aussi son expérience, qui se révèle elle-même infinie. Non une expérience qui dure infiniment, mais un basculement infini, qui ne cesse ses allers-retours, faisant naître chez le sujet les conditions de sa propre réitération par un travail actif de la subjectivité, ainsi que le note B. Saint-Girons:

Le beau se suffit à lui-même et "subsiste" au paradis éternel des idées, indépendamment de toute reconnaissance; mais le sublime, lui, ne fait qu'exister dans la fragilité de ce qui doit se perpétuer ailleurs qu'en lui-même pour survivre: il m'exige et m'entame, faisant appel à la subjectivité dans ce qu'elle a de plus mystérieux ${ }^{33}$.

On n'a sans doute pas prêté suffisamment d'attention à cette courte phrase d'Alexis Philonenko, en commentaire de sa traduction de Kant, qui est à entendre dans toute sa profondeur: "Le sublime est, phénoménologiquement décrit, une infinité qui se redouble sans cesse ${ }^{34}$. "Pas de dualité, mais une duplicité qui, mise en mouvement, se répète, se redouble à l'infini. Le basculement semble donc être la forme dynamique traduisant au mieux l'énergie présente tant dans l'expérience esthétique que dans le concept qui la représente et la juge réflexivement. Le sublime, à la différence du beau, traduit des émotions qui ne sont pas suscitées par de pures formes, mais également par les Idées que ces formes amènent à la réflexion. Aussi s'agiraitil, dans le sublime, de présentations sensibles renvoyant négativement au supra-sensible, soit d'Idées de la raison à même de susciter en nous une émotion esthétique: "le sublime authentique [...] ne concerne que les Idées de la raison, qui, bien qu'aucune présentation adéquate n'en soit possible, sont néanmoins rappelées en l'esprit et ravivées de par cette inadéquation même, dont une présentation sensible est possible ${ }^{35}$ ». En s'éloignant de la théorie kantienne, nous sommes capables de nous imaginer une émotion esthétique "intellectuelle", qui serait liée à la représentation d'un concept, quel qu'il soit. Et pourquoi l'idée même du sublime ne serait-elle pas justement capable d'en susciter le sentiment, en étant prise elle-même comme objet d'un jugement esthétique? Si le sentiment du sublime nous amène réflexivement à son concept, l'idée du sublime peut-elle entretenir en nous son expérience? Comme le signale Baldine Saint-Girons, il ne faut «jamais réduire le sublime à son principe ou à sa conséquence, mais penser toujours

33. Ibid., p. I02-103.

34. A. Philonenko, in I. Kant, Critique de la faculté de juger, op. cit., p. I36, note I. Nous soulignons.

35. Ak, V, 245. Trad., p. I20. 
l'un comme engendrant l'autre dans une causalité proprement circulaire ${ }^{36}$ ». Le propre du sublime serait alors «d'engendrer le sentiment et la pensée qui le découvrent ${ }^{37}$ ». Quoi qu'il en soit, le basculement semble pouvoir s'opérer dans les deux sens, et lie, d'une manière propre au sublime, concept et expérience. En d'autres termes, le basculement répété annonce une circularité où l'idée du sublime, naissant d'une réflexivité sur son sentiment, devient ellemême la cause de ce sentiment... Cet aller-retour infini du vécu et de sa conscience, réitérant le sublime ou suggérant à tout le moins sa future répétition, enregistre son événement dans une certaine durée.

Le sublime ne peut dès lors plus être considéré comme un événement esthétique tel que nous avions pu le décrire, pure saillie ou brèche dans le temps. S'il y a bien une dimension d'événement au plus intime du concept, c'est dans son articulation même, dans la façon dont il s'inscrit dans la temporalité et la remet en jeu, en mouvement. En effet, l'expérience du sublime semble correspondre à un suspens de toute durée: dans la fulgurance et l'éclair du sublime, l'homme est saisi par une tension qui le paralyse. Novalis aura une formule qui traduit bien cette idée: "leur observateur aux sens aiguisés [des statues datant de l'âge d'or de l'humanité] est recouvert d'une écorce minérale semblant croître vers l'intérieur. Le sublime produit de l'effet en pétrifiant [Das Erhabne wirkt versteinernd ${ }^{38}$ ". Changeant en pierre, le sublime engendre, pour une part, une expérience du hors-temps, un étonnement sortant l'homme de la trame du quotidien pour lui permettre d'embrasser en une intuition l'horizon de sa destination rationnelle. Mais simultanément, l'homme prend conscience de sa finitude et de son inscription dans la contingence de l'Histoire. Ce n'est que parce qu'il ne peut tout comprendre (sublime mathématique), parce qu'il peut être détruit par des forces qui le dépassent (sublime dynamique), que l'homme découvre par contraste la permanence de sa dignité en tant qu'être moral. Le sublime correspond à l'expérience pour l'homme de son inscription dans une temporalité double: être historique et transcendantal, il se découvre soumis à la finitude d'un temps qui le conduit à la mort et lui ouvre simultanément une marge d'action, lui fait prendre conscience d'un certain intervalle où peut s'exercer la puissance de sa raison. À notre tour, nous pourrions faire «basculer» notre formule pour avancer qu'une esthétique de l'événement est en jeu dans le sublime. C'est-à-dire une certaine expérience esthétique du temps, opérée dans la dynamique et la tension propres au paradoxe de l'événement. Un événement dès lors sans objet, non plus événement de quelque chose, mais qui ne fait plus rien arriver si ce n'est lui-même, comme a pu le

36. B. Saint-Girons, Fiat Lux, une philosophie du sublime, Paris, Quai voltaire, I993, p. $5 \mathrm{I} 8$.

37. Ibid., p. I2.

38. Novalis, Les disciples à Sä̈s, trad. fr. A. Dumont, Les Belles Lettres, Paris, 20I4, p. 183 . 
décrire J.-P. Curnier: «En somme, l'événement c'est la valeur du nouveau sans l'objet, sans l'encombrement de l'objet ${ }^{39}$." Expérience esthétique de l'événement comme événement, le sublime ne prend réellement sens que dans son expérience, qui s'accompagne réflexivement d'une signification conceptuelle. Pour reprendre les mots de M. Baudin cette expérience sera

une rencontre dans l'éprouvé avec un événement qui, au lieu de faire trauma et effraction, apporte au contraire la plénitude, le cadeau d'un accord, fugace mais parfait, entre la source de l'événement et soi-même. [...] Exstase, stase "hors limite", état sub ou extra-liminal que ce moment où, sous l'effet d'un attracteur "étrange" a lieu le dépassement de soi, qui pousse les limites vers le sublime $^{40}$.

Que le sublime puisse nous conduire à penser le temps, par le rapport qu'il entretient à l'événement, cela ne doit pas nous étonner outre mesure lorsqu'on lit les développements de Kant sur ce qu'il nomme les "Idées esthétiques". Ces dernières, qui trouvent également place dans l' "Analytique du sublime", incarnent sans doute l'ultime paradoxe de la Critique kantienne, comme l'a éminemment bien montré J.-P. Larthomas:

Car c'est, par rapport à la théorie kantienne des Idées, un paradoxe d'abord terminologique, provoquant comme une contradiction in adjecto. Comment une Idée, qui est par définition un concept non schématisable, pourrait-elle se faire intuitive et sensible, selon le sens encore très proche du grec que conserve toujours l'adjectif "esthétique" chez Kant ?+1

Le philosophe de Königsberg définit l'Idée esthétique comme la possibilité d'une intuition (de l'ordre de l'imagination) reconduisant aux Idées de la raison, impulsant un élan aux facultés de l'esprit: c'est une « représentation de l'imagination, qui donne beaucoup à penser, sans qu'aucune pensée déterminée, c'est-à-dire de concept, puisse lui être adéquate, et que par conséquent aucune langue ne peut complètement exprimer et rendre intelligible $^{42} »$. Cette définition fait écho au mode opératoire du sublime, lui qui ouvre notre imagination au supra-sensible sans nous délivrer de concept déterminé de celui-ci mais en nous faisant "penser", en un sens proche de celui employé par Descartes ${ }^{43}$. Le sublime semble avoir partie liée avec l'Idée esthétique, comme jugement ou expérience de cette dernière, ce qui justifie entièrement la place de ce développement au $\$ 49$ de l'«Analytique du

39. J.-P. Curnier, «Esthétique de l'événement ", Lignes I996, 3 (nº 29), p. Io9.

40. M. Baudin, "L'expérience du sublime», op. cit.

41. J.-P. Larthomas, "Le paradoxe de l'Idée esthétique ", in M. Frank, J.-P. Larthomas et A. Philonenko, Sur la Troisième Critique, op. cit., p. 49.

42. Ak, V, 3I4. Trad., p. 2 I3.

43. R. Descartes, Principes de la philosophie, I, 9: «Par le mot penser, j'entends tout ce qui se fait en nous de telle sorte que nous l'apercevons immédiatement par nous-mêmes; c'est pourquoi non seulement entendre, vouloir, imaginer, mais aussi sentir, est la même chose ici que penser. » 
sublime ». Les Idées esthétiques «tendent pour le moins à quelque chose qui se trouve au-delà des limites de l'expérience et cherchent ainsi à s'approcher des concepts de la raison ${ }^{44}$ ", stimulant l'imagination dans sa capacité à créer « une autre nature [...] à partir de la matière que la nature réelle lui donne » afin de «transformer l'expérience quotidienne ${ }^{45}$ ».

\section{Une poétique du sublime}

Ces différents éléments d'analyse nous ont permis de comprendre la structure formelle du sublime, tel qu'il est développé par Kant. Nous avons précisé la forme du mouvement animant l'expérience du sublime, son rapport spatio-temporel à l'idée de limite, la place que prend le paradoxe dans l'économie du concept. Les lignes de force ainsi dégagées se sont révélées essentielles pour comprendre la temporalité de cette expérience singulière, indissociable par ailleurs d'une certaine pensée du temps lui-même, à l'œuvre dans le jugement esthétique du sublime. Plus globalement, il semble que l'expérience du sublime soit liée intimement à un sens dépendant d'Idées esthétiques auxquelles nous conduit réflexivement l'expérience du sublime. Mais cette formalisation du sublime ne nous dit encore rien des façons dont l'événement peut être repensé dans sa dimension paradoxale, de la nature de ces Idées esthétiques: elle ne fait que fonder la possibilité de cette entreprise. C'est par les formes concrètes pouvant susciter le sublime que pourra se préciser le sens surgissant de cette expérience de l'événement.

Pour Kant, c'est principalement la poésie qui sera susceptible de présenter de telles Idées esthétiques capables de nous rendre sensible à ce qui dépasse notre expérience, ou d'élever à l'intelligible ce qui constitue le fondement de notre vie sensible:

Le poète ose donner une forme sensible [versinnlichen] aux idées de la raison que sont les êtres invisibles, le royaume des saints, l'enfer, l'éternité, la création... [soit l'absence de nouveauté, donc d'événement, soit son apparition] etc., ou bien encore à des choses dont on trouve au vrai des exemples dans l'expérience, comme la mort, l'envie et tous les vices, ainsi que l'amour, la gloire... etc., mais en les élevant alors au-delà des bornes de l'expérience, grâce à une imagination, qui s'efforce de rivaliser avec la raison dans la réalisation d'un maximum, en leur donnant une forme sensible dans une perfection dont il ne se rencontre point d'exemple en la nature; et c'est en la poésie que la faculté des idées esthétiques peut donner toute sa mesure ${ }^{46}$.

Ce passage, aussi brillant que renversant, fonde le lien intime que le sublime peut entretenir avec l'art et, plus spécifiquement, avec l'opération de poétisation. Comme le précise J.-P. Larthomas, «on comprend ce privilège: la poésie œuvre dans l'élément du langage, donc en relation (même libre) avec

44. $A k, \mathrm{~V}, 3 \mathrm{I} 4$. Trad., p. 2 I4.

45. $A k, \mathrm{~V}, 3 \mathrm{I} 4$. Trad., p. 2 I 3 .

46. Ak, V, 3 I4. Trad., p. 2I4. Nous soulignons. 
l'entendement. "La poésie est l'art de conduire un libre jeu de l'imagination comme une activité de l'entendement" $\left(\mathbb{S}_{5} \mathrm{I}\right)^{47}$.» À l'endroit précis où Kant introduit un appel de note renvoyant à la sentence d'Isis, le philosophe nous explique la double possibilité offerte au poète d'amener l'esprit jusqu'à l'Idée rationnelle au moyen d'attributs esthétiques et/ou, inversement, de toucher notre sensibilité par la mobilisation d'Idées de la raison (son exemple porte sur l'évocation de l'idée de vertu). Une autre notion, celle de génie, se montrant surtout "dans l'expression d'Idées esthétiques ${ }^{48}$ ", est au centre de cette interaction, représentant comme l'adéquation dans une œuvre entre présentation sensible et représentation supra-sensible:

Ainsi le génie consiste proprement dans un heureux rapport, qu'aucune science ne peut enseigner et qu'aucun labeur ne permet d'acquérir; ce rapport est celui en lequel d'une part on trouve les Idées se rapportant à un concept donné et d'autre part l'expression qui leur convient, et par laquelle la disposition subjective de l'âme ainsi suscitée, comme accompagnant un concept, peut être communiquée à autrui ${ }^{49}$.

Ce que nous jugeons sublime, c'est donc bien une représentation de l'imagination qui

donne par elle-même bien plus à penser que ce qui peut être compris dans un concept déterminé, et qui par conséquent élargit le concept lui-même esthétiquement d'une manière illimitée, l'imagination est alors créatrice et elle met en mouvement la faculté des Idées intellectuelles (la raison) ${ }^{50}$.

Les Idées esthétiques sont développées par le génie qui incarne un moment de l'art où s'opère un juste rapport entre idées et sentiments, les représentations de l'imagination et celles de la raison. Le sublime nous amène, par là, à réfléchir les idées, les concepts eux-mêmes d'une façon certes différente de celle de l'entendement ou de la raison pratique, mais qui nous dévoile d'autres choses, notamment par une expérience sensible de ces concepts. «Il faut que les sensations poétiques "ne permettent pas seulement un sentiment sensible, mais aussi la réflexion sur la forme de ces modifications des sens" $(\$ 42)[\ldots]$ Il s'agit moins d'une conscience réflexive que d'une réflexion du sentiment, entraîné par la forme de l'œuvre ${ }^{51}$ ", note avec justesse J.-P. Larthomas. Ce lien du concept à son vécu sensible est consacré par le sublime, dont l'expérience elle-même suit totalement l'idée: ainsi la mise à nu d'une certaine nature paradoxale de la temporalité n'est pas que le résultat ou l'objet du sublime, elle est toujours déjà à l'œuvre dans l'expérience du sublime qui ne la dévoile que parce qu'elle s'organise elle-même comme le

47. J.-P. Larthomas, "Le paradoxe de l'Idée esthétique ", op. cit., p. 57.

48. Ak, V, 317. Trad., p. 2 I 8.

49. Ak, V, 317. Trad., p. 217-2I8. Nous soulignons.

50. Ak, V, 315 . Trad., p. 2 I 4.

51. J.-P. Larthomas, «Le paradoxe de l'Idée esthétique », op. cit., p. $5^{8 .}$ 
sentiment d'un basculement temporel. Par ailleurs, il ne faudrait pas croire que les Idées esthétiques développées par le sublime ne concerneraient que le temps et notre rapport à lui. Si nous insistons sur cette dimension, c'est pour montrer l'intrication nécessaire de l'expérience avec un sens réflexif dans le concept, abordé ici sous le prisme de l'événement. Mais ce que nous fait «penser» le sublime ne concerne pas que "l'éternité ", d'autres principes métaphysiques sont intimement liés à cette expérience qui va jusqu'à nous dévoiler par l'entremise de la poésie «les idées de la raison que sont les êtres invisibles, le royaume des saints, l'enfer». Si l' "Analytique du sublime» nous révèle en définitive qu' 'il existe en fait deux façons (modus) d'agencer l'exposé de ses pensées, dont l'une s'appelle une manière (modus aestheticus) et l'autre une méthode (modus logicus) ", la première n'ayant «d'autre mesure que le sentiment de l'unité dans la présentation ${ }^{52}$ » et seule valable pour les beaux-arts, c'est précisément parce que sensible et supra-sensible s'engendrent mutuellement dans l'expérience du sublime.

Pour citer encore Larthomas: "Grâce à la re-composition du matériau de la sensation, la forme poétique peut traiter la nature "pour ainsi dire comme schème du supra-sensible". De la forme, la pensée réfléchissante s'élève donc à l'Idée $e^{53}$. " Le rapport particulier du sublime à la poésie n'est pas en contradiction brutale avec l'idée kantienne que le sublime ne réside en aucun objet, ne pouvant être trouvé qu'en notre esprit. Il ne s'agit pas de dire que tel ou tel poète, tel ou tel poème, serait sublime. Mais le travail de "poétisation» du réel s'apparente à une sublimation, présentant, par son procès langagier, le mouvement du sublime dans notre subjectivité. Kant l'avance explicitement: pour que survienne l'expérience sublime, il s'agit d'accorder sa perception à l'ouverture de l'événement, travail esthétique qui est précisément celui de la poésie:

Il faut arriver à voir l'océan seulement, comme le font les poètes, selon le spectacle qu'il donne à l'œil, soit lorsqu'il est contemplé au repos tel un clair miroir d'eau qui n'est limité que par le ciel [sublime mathématique] et, lorsqu'il est agité, soit comme un abîme menaçant de tout engloutir, qu'il nous est quand même possible de trouver sublime [dynamique] ${ }^{54}$.

Par là peut-être le sublime échappe-t-il à l'imprésentable, à l'indicible hypostasié en un absolu transcendant: dans l'immanence d'une forme poétique s'engendre un sublime qui s'invente en même temps qu'il performe les représentations des hommes, notamment celle du temps.

Pour esquisser un prolongement à cette conception, nous pourrions songer au projet romantique d'Iéna, héritier de la troisième Critique, et qui dans son ensemble vise précisément à maintenir la tension dégagée dans

52. Ak, V, 3I8-3I9. Trad., p. 220.

53. J.-P. Larthomas, "Le paradoxe de l'Idée esthétique », op. cit., p. 54.

54. Ak, V, 270. Trad., p. I 55. Nous soulignons. 
l'événement: faire une place à l'Histoire, à l'inscription de l'homme dans la contingence d'un ici et d'un maintenant, tout en conservant la réflexivité transcendantale et ses concepts, hérités en droite ligne de la pensée kantienne. Et c'est une grande part de la modernité poétique, au moins jusqu'à Proust, qui hérite de cette question. L'inscription de l'art romantique sous le signe du sublime tient sans doute bien moins au développement d'une esthétique du grandiose ou de l'héroïsme qu'à la mise en forme d'un questionnement métaphysique portant, entre autres, sur le rapport de l'homme à son histoire, questionnement cristallisé dans une poiétique de l'événement création mais aussi épreuve de ce dernier - développée chez divers auteurs tels que Nerval, Baudelaire, Proust ${ }^{55}$, pour ne citer qu'eux. Il ne s'agit donc pas d'échanger une abstraction autonome pour une autre: l'événementbasculement n'est pas, semble-t-il, qu'un autre avatar d'un événement-surgissement, mais renvoie à un travail précis de l'imagination qui s'incarne de façon singulière chez chacun de ces auteurs. Leur intérêt ne résiderait pas pour autant dans une illustration de la théorie développée ici : ces poétiques poursuivent plutôt la théorie et, chacune singulièrement, contribuent à travailler ce paradoxe de l'événement dont le sublime concentre l'expérience. Ce n'est qu'en poésie que nous pouvons dire, avec René Char, «l'éclair me dure », ce court fragment de La Parole en Archipel qui reprend l'image si chère au Pseudo-Longin - l'éclair - pour condenser en quelques mots le paradoxe structurant la temporalité de l'expérience du sublime.

En conclusion, le sublime est événement esthétique non parce qu'il créerait abstraitement "du sens », mais parce qu'il incarne simultanément le jugement et l'expérience d'une représentation de l'imagination qui amène l'esprit non à connaître des Idées de la raison, mais à les penser, ouvrant l'imagination à une infinité de possibles non déterminables d'avance sous

55. Nous renvoyons aux excellentes analyses d'A. Simon qui montre que dans l'idée proustienne de L'adoration perpétuelle, «l'Instant sublime» opère comme un pont entre Temps perdu et temps retrouvés, qui "dans leur antagonisme, permettent la "résurrection" du narrateur et la promesse de l'œuvre littéraire " (A. Simon, "Les “Temps retrouvés” ", Revue d'histoire littéraire de la France, 2003, 4 (vol. I03), p. 9I7.) Proust rend d'ailleurs compte d'un paradoxe correspondant à celui que nous avons pu dégager plus haut: "Je découvrais cette action destructrice du Temps au moment même où je voulais entreprendre de rendre claires, d'intellectualiser dans une œuvre d'art, des réalités extra-temporelles» (T. R., IV, 508). Chez Nerval aussi se rejoue sans cesse le rapport du narrateur à la temporalité sur un mode paradoxal, semblable à celui que nous avons dégagé dans l'expérience du sublime. Si le temps importe chez Nerval, il peut néanmoins être pensé comme un miroir sans durée réelle où le moi se reflète dans son propre passé. Tout en intégrant de manière forte sa propre histoire au récit, Nerval développe un langage anhistorique où l'événement s'annule comme événement, tout en trouvant une signification plus large — paradoxalement comme représentation même du temps — dans le hors-champ du rêve, défini comme "phénomène d'espace analogue à celui du temps qui concentre un siècle d'action dans une minute de rêve" (Aurélia). Voir également l'étude de P. Destruel sur le rapport de Nerval à la temporalité: P. Destruel, L'écriture nervalienne du temps, Paris, Nizet, 2004. 
une règle. Le sublime serait dès lors au départ d'une esthétique de l'événement, une expérience du temps (mais également d'autres notions structurant notre rapport au monde). Cette expérience se déploierait dans une duplicité paradoxale, faisant appel, depuis le sensible, à une reformulation réflexive du principe de temporalité pouvant trouver place, par exemple, en poésie. En effet, notre analyse de l'événement du sublime ne nous a permis que de dégager la structure formelle de cette expérience. Encore s'agirait-il de voir quelles Idées esthétiques pourraient être développées en poésie, sous le signe du génie, et en quoi elles travailleraient nos représentations, par l'intermédiaire du jugement esthétique du sublime. Pour le dire avec J.-P. Larthomas, "l'œuvre d'art n'a pas le privilège surhumain sur une sorte de "science intuitive" du troisième genre. Mais elle a celui d'informer la sensibilité et le "sens commun" esthétique, pour les enrichir de résonances dans l'effort vers la pensée qui questionne ${ }^{56}$.» Dans les différentes mises en forme poétiques de l'expérience du temps, que nous jugerions sublimement, nous pourrions donc identifier précisément en quelle façon, de quelle manière, l'Idée esthétique nous donnerait «à penser en plus d'un concept bien des choses indicibles, dont le sentiment anime la faculté de connaissance et qui inspire à la lettre du langage un esprit ${ }^{57}$ ».

\section{Bibliographie sélective}

Badiou, Alain. L'être et l'événement, Paris, Le Seuil, 1988.

Baudin, Marianne. "L'expérience du sublime", Topique, 2009, 4, n I09, p. I77-I 87.

Calori, François, Michaël Foessel et Dominique Pradelle. De la sensibilité: les esthétiques de Kant, Rennes, Presses Universitaires de Rennes, 2014.

Carrive, Paulette. «Le Sublime dans l'esthétique de Kant ", Revue d'histoire littéraire de la France, $\mathrm{n}^{\circ}$ I : Le Sublime, I986, OI ; I986, O2, p. 7I-85.

Chedin, Olivier. Sur l'esthétique de Kant et la théorie critique de la représentation, Paris, Vrin, I982.

Courtine, Jean-François, Michel Deguy, et al. Du sublime, Paris, Belin, 2009.

Curnier, Jean-Paul. «Esthétique de l'événement », Lignes (n² 29), I 996, 3, p. IO6-I2I.

Deleuze, Gilles. Différence et répétition, Paris, PUF-Épiméthée, I968; Logique du sens, Paris, Minuit, I969.

Diano, Carlo. Forma ed Evento. Principii per una interpretazione del mondo Greco, Venezia, N. Pozza, I952.

Frank, Manfred, Jean-Paul Larthomas et Alexis Philonenko. Sur la Troisième Critique, Combas, Éditions de l'Éclat, I994.

Gueguen Haud. «Du possible à l'événement: essai de typologie à propos de l'événement et de la contingence ", Nouvelle revue de psychosociologie ( ${ }^{\circ}$ I9), 2015 , I, p. 79-92.

Hegel, G. W. F. Esthétique, B. Timmermans et P. Zaccaria (dir.), Paris, Le Livre de poche, I997, vol. I.

56. J.-P. Larthomas, «Le paradoxe de l'Idée esthétique », op. cit., p. 66.

57. Ak, V, 3 I6. Trad., p. 2 I 7. 
Höffe, Otfried (Hrsg.). Kritik der Urteilskraft, Berlin, Akademie Verlag, 2008.

Kant, Immanuel. Kritik der Urteilskraft, hrsg. von der Königlich Preußischen Akademie der Wissenschaften $(A k)$, Bd. V, Berlin, I908; Kant, Emmanuel, Critique de la faculté de juger, trad. fr. A. Philonenko, Paris, Vrin, 2000.

Lyotard, Jean-François. Leçons sur l'Analytique du sublime: Kant, critique de la faculté de juger, [Sections] 23-29, Paris, Galilée, I99I.

Peyrache-Leborgne, Dominique. La poétique du sublime, de la fin des Lumières au Romantisme, Paris, Champion, 1997.

Pries, C. (Hrsg.). Das Erhabene. Zwischen Grenzerfahrung und Größenwahn, Weinheim, VCH, I989.

Romano, Claude. L'événement et le monde, Paris, PUF-Épiméthée, I998; L'événement et le temps, Paris, PUF-Épiméthée, I999.

Saint Giron, Baldine. Fiat Lux: une philosophie du sublime, Paris, Quai voltaire, I993; Le sublime de l'Antiquité à nos jours, Paris, Desjonquères, 2005 ; "Le sublime » in CD Universalis [vidéo sur CD-ROM], Paris, Encyclopaedia universalis, 2006.

Salvesen, Christopher. «Aspects of the Romantic Sublime», Charles Lamb Bulletin, $\mathrm{n}^{\circ} 50, \mathrm{I} 985, \mathrm{p} .37-5 \mathrm{I}$.

Schiller, Friedrich. Du Sublime, trad. fr. A. Régnier, Arles, Sulliver, 2005.

Simon, Agathe. "Proust. L'instant et le sublime», Revue d'histoire littéraire de la France (vol. I03), 2003, 4, p. 86I-887; "Les “Temps retrouvés” ", idem, p. 889-9I7.

Souriau, Étienne. "Le sublime", Revue d'esthétique n I9, I966, p. 266-289.

Weiskel, Thomas. The romantic Sublime. Studies on the Structure and Psychology of Transcendance, Baltimore - London, The Johns Hopkins University Press, I 976. 